\title{
HOLOGRAPHY WITH NON-COHERENT LIGHT
}

\author{
Byung Jin CHANG \\ The Enviroumental Research Institute of Michigan, \\ P.O. Box 618, Ann Arbor, Michigan 48107, USA \\ and \\ The University of Michigan, \\ Ann Arbor, Michigan 48107. USA
}

Received 18 October 1973

\begin{abstract}
A new technique of making holograms with non-coherent light has been demonstrated using continuousspectrum white light zirconium are source. Such a technique, employing diffraction gratings as beam splitters and beam deflectors. allows casy control of the modulation transfer function so as to produce low-pass. band-pass or high-pass characteristics by simply changing the position of either one of the gratings or the tecording plane.
\end{abstract}

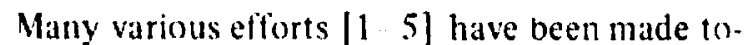
ward the production of high-quality holographic image with light of low coherence. The purpose of these efforts has been either (1) the improvement of the resolution from an available limited-coherence light source, in order to adapt such potentially usetil sources as multi-line gas lasers. injection lasers, light emitting diodes, dye lasers, cathode ray tubes, gas discharge tubes, or $\mathrm{X}$-ray sources to holographic systems, or (2) the avoidance of highly coherent light, which aggravates the artifact problems.

Leith and Upatnieks [2] demonstrated a configuration that recluced off-axis coherence requirements to those of in-line holograms. Later Katyl [4] demonstrated that coherence requirement may be reduced further. However, all previous techniques employed lenses and prisms or lenses and gratings. and thus are restricted in the aperture available. In such systems the modulation transfer function of hologram cannot be controlled easily.

We have devised systems employing diffraction gratings as beam splitters and beam deflectors: one of these configurations is shown in fig. 1. Such systenı, as shown in previous analysis [5], have no limitations on object size, hologram size, or number of fringes generated. Moreover, in such systems we

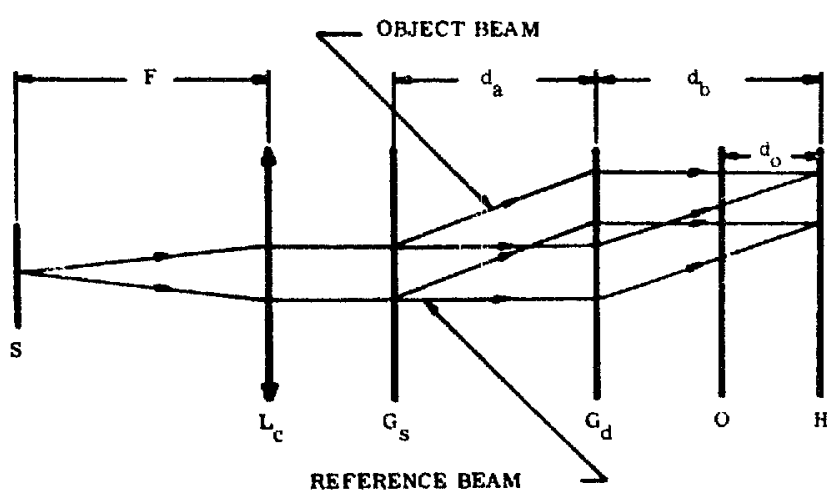

Fig. 1. Ifolograpinic system based on grating-interferometer. $S$ is the source, $L_{c}$ is a colliminating lens of focal length $F$. $G_{\mathrm{S}}$ is the grating used as beam splitter, $G_{d}$ is the grating used as deflector, $O$ is the object plane and $H$ is the hologram recording plane.

ean adjust the modulation transfer function so as to produce low-pass, band-pass or higi-pass chatacterislics by simply changing the position of either the grating $G_{\mathrm{s}}$ or the grating $G_{\mathrm{d}}$; also the same effects can be obtained by changing the position of recording plane $H$. Even though, in practice, there is a limitation on attainable resolution from a source of given coherence, the coherence requirement can be reduced con- 
siderably. The elassical limit of hologram resolution for a given coherence is no longer true in this new system. This technique can in principle be applied in such non-visible waves as electron beam, acoustic wave, microwave, or X-ray.

The important property of the grating-based interferometers is that they form broad-source, achromatic fringes; even with an ordinary flash light very high contrast fringes can be generated. Therefore, this system can use broad-source white light in both the processes of hologram construction and reconstruction. with a resultant reduction of the artifact noise.

Here we report some experiments on hologram formation and reconstruction with non-laser light sources. The detailed theoretical analysis and further discussions on various applications will be reported later.

The system used is shown in fig. 1, where gratings $G_{s}$ and $G_{d}$ have the same spatial frequency. If $w e$ neglect the effect of source size and assume uniform intensity distribution over the spectrum range of $\lambda_{0}-\Delta \lambda \leqslant \lambda \leqslant \lambda_{0}+\Delta \lambda$, the modulation transfer function is given by

$\left.H(j)=\sin c\left(i-d_{0} \hat{p}^{2}+\kappa_{0}\right) \Delta \lambda\right]$.

where

$\AA_{0}=\left(d_{b} \quad d_{3} \mid f_{0}^{2}\right.$.

$F_{0}$ is the spatial frequency of the gratings $G_{\mathrm{s}}$ and $G_{\mathrm{d}}$, $\nu_{0}$ is hologram object distance and $\beta$ is spatial frequency of the object. Letting $\beta=d_{0} \beta^{2} \Delta \lambda$ and $K_{0}=$ $\kappa_{0} \Delta \lambda$, we have plotted $H(\bar{\beta})$ as a function of $\bar{\beta}$ for various values of $K_{0}$ in fig. 2 .

Fig. 3 shows the effect of source spectral width on resolution, and how adjustment of $\bar{K}_{0}$ can compensate for the broadened source spectrum. At the top is shown the original object, with letters $0.5 \mathrm{~mm}$ in heigh:. Also, we show images from holograms made with the green line of the $\mathrm{Hg}$ source (the $\mathrm{Hg}$ series) and with the white light of the zirconium arc source the $Z i$ series?. The value of $K_{0}$ increases from a to $c$. For $\mathrm{Hg}(a)$, the value of $K_{0}$ is less than 0 . which is a rather unfavorable case. For $\mathrm{Hg}(b), K_{0}=0$, which, as described in ret. $|5|$. gives a passband equivatent to conventional, in-line holography. In $\mathrm{Hg}(\mathrm{c})$,

\footnotetext{
$\because$ A similar equation was given in ref. [5], but the exact derivation uill be given in a future paper.
}

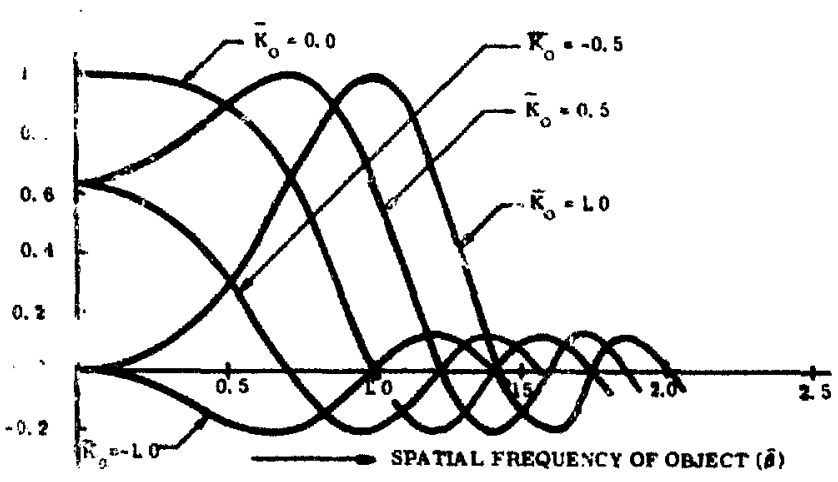

Fig. 2. Modulation transfer function as a $f$ ction of $\bar{\beta}$ for various values of $\kappa_{0}$.

$K_{0}$ is about 1 , which yields a decidedly high-pass characteristic, at the expense of loss of lower spatial frequencies, and a resulting poor signal to noise ratio.

For the $\mathrm{Zi}$ series, we have holograms formed with continuous-spectrum white light. $Z i(a)$ shows the $\bar{K}_{0}=0$ case. In $\mathrm{Zi}(\mathrm{b})$, we have $\bar{K}_{0}$ about 0.6 , wich we judge to be a good compromise between edge sharpening and preservation of the higher spatial frequency content. $\mathrm{Zi}(\mathrm{b})$ is overall better than $\mathrm{Zi}(\mathrm{a})$. and shows that this technique permits rather good holograms to be produced in completely white light. For $\mathrm{Zi}(\mathrm{c}), K_{0}=1$, and the comments made about $\mathrm{Hg}(\mathrm{c})$ apply here.

From these experimental results, we conclude that the attainable control of the modulation transfer function can increase the hologram resolution considerably, and that as long as a light source having wide spectrum is of reasonably small size, it could find useful applications in holography.

Thanks is given to the National Science Foundation (Grant No. 31474) for their support of this work, and to E.N. Leith for his guidance and encouragement.

\section{References}

[1] H.R. Worthington, Jr., J. Opt. Soc. Am. 56 (1966) 1397.

[2] E.N. Leith and J. Upatnicks, J. Opt. Soc. Am. 57 (1967) 975.

[3] O. Bryngdahl and A. Lohmann, J. Op. Soc. Am. 60 (1970) 281 .

[4] R.H. Katyl, Appl. Opt. 11 (1972) 1241,1248 ind 1255.

[S] F.N. Leith and B.J. Chang, Appl. Opt. 12 (1973) 1957. 


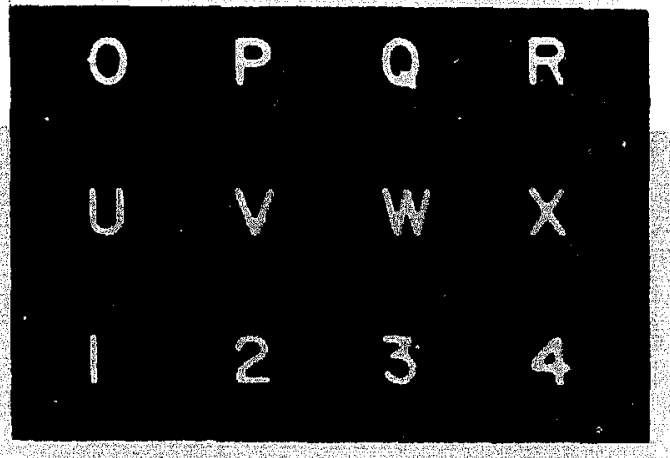

original

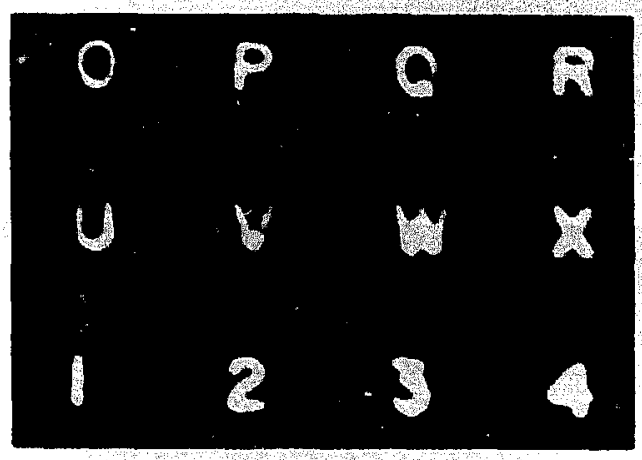

$\mathrm{Hg}(\mathrm{a})$

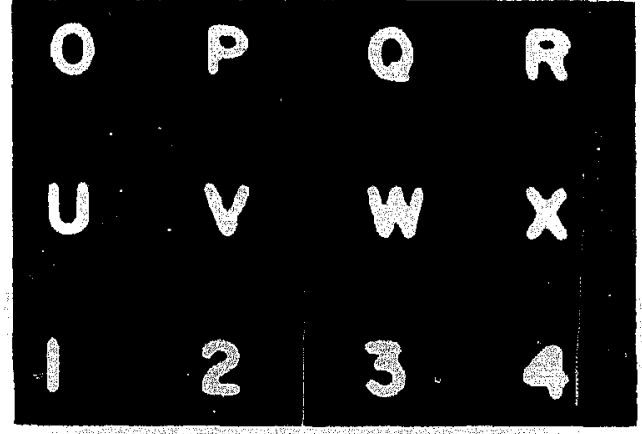

$\mathrm{Hg}(\mathrm{b})$

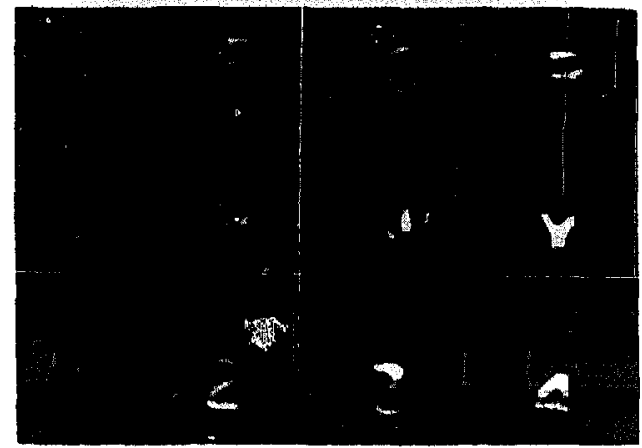

$\operatorname{Hg}(\mathrm{c})$

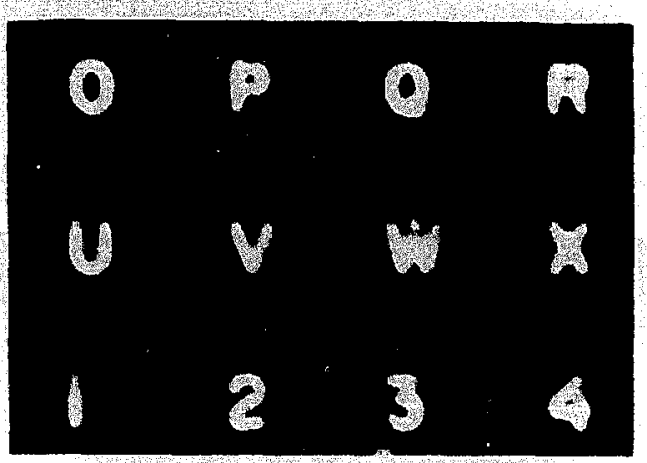

$21(a)$

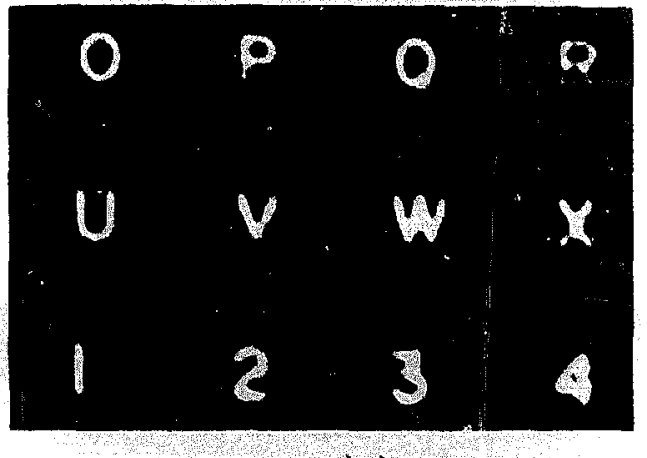

$\mathrm{Zi}(\mathrm{b})$

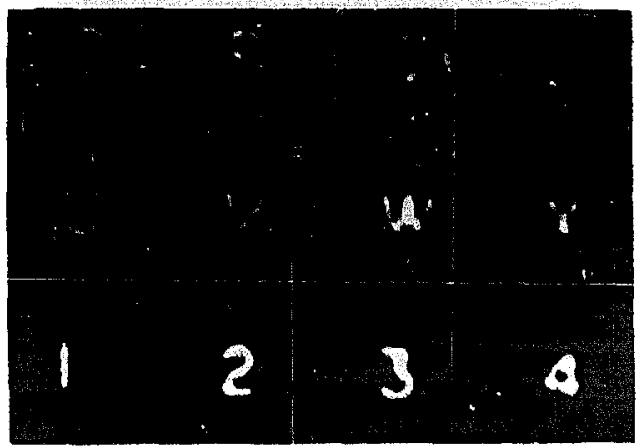

$7 \mathrm{i}$ (c)

Fig. 3. Images produced from holograms made in the system shown in fig. 1 to show the effect of spectrum width of light source and the effect of the modulation transfer function on the resolution of holographic imagery. $f_{0}$ is 200 lines/mm, $d_{\mathrm{a}}=d_{\mathrm{b}}=55 \mathrm{~mm}$, $d_{0}=25 \mathrm{~mm}$ and the height of letters is $0.5 \mathrm{~mm}$. The source size (diameter of pinhole) is 150 micrnns and $F=240 \mathrm{~mm}$. 